\title{
LEVOMEPROMAZINA E ACEPROMAZINA NO BLOQUEIO DA ARRITMIA INDUZIDA PELA ADRENALINA EM CÃES ANESTESIADOS PELO HALOTANO
}

\author{
LEVOMEPROMAZINE AND ACEPROMAZINE TO BLOCKADE THE ARRHYTHMIA \\ INDUCED BY EPINEPHRINE IN DOGS ANESTHETIZED WITH HALOTHANE
}

\author{
Márlis Langenegger de Rezende ${ }^{1}$ Anderson Farias ${ }^{1}$ Aline Adriana Bolzan ${ }^{1}$ \\ Wagner Luis Ferreira ${ }^{1}$ Elzylene Léga ${ }^{1}$ Newton Nunes ${ }^{2}$
}

RESUMO

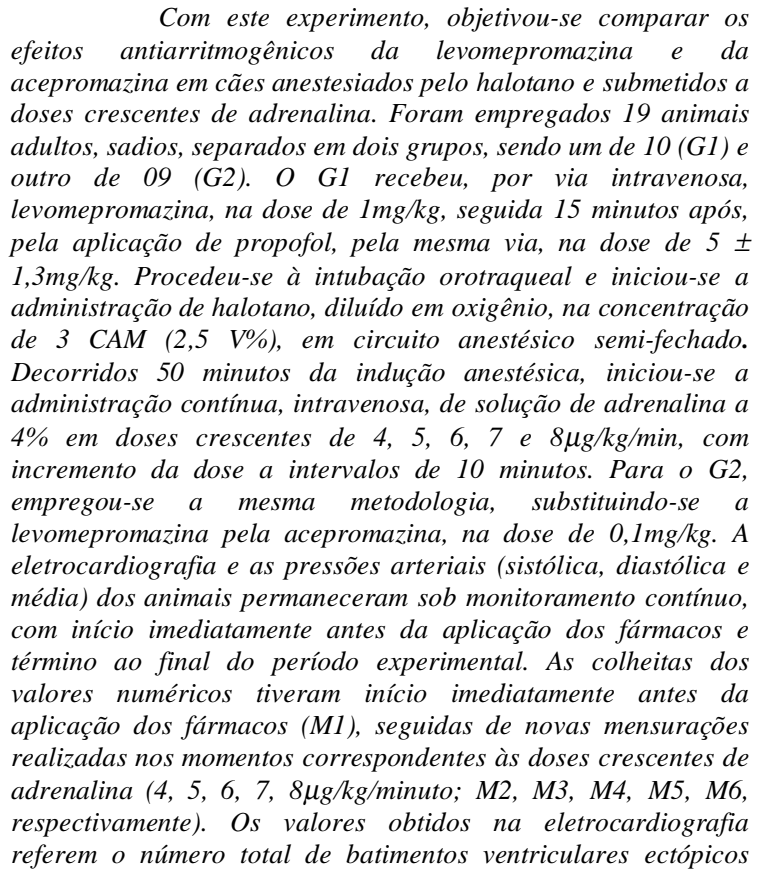

Com este experimento, objetivou-se comparar os efeitos antiarritmogênicos da levomepromazina $e \mathrm{da}$ acepromazina em cães anestesiados pelo halotano e submetidos a doses crescentes de adrenalina. Foram empregados 19 animais adultos, sadios, separados em dois grupos, sendo um de $10(G 1) e$ outro de 09 (G2). O G1 recebeu, por via intravenosa, levomepromazina, na dose de $1 \mathrm{mg} / \mathrm{kg}$, seguida 15 minutos após, pela aplicação de propofol, pela mesma via, na dose de $5 \pm$ $1,3 \mathrm{mg} / \mathrm{kg}$. Procedeu-se à intubação orotraqueal e iniciou-se a administração de halotano, diluído em oxigênio, na concentração de 3 CAM (2,5 V\%), em circuito anestésico semi-fechado. Decorridos 50 minutos da indução anestésica, iniciou-se a administração contínua, intravenosa, de solução de adrenalina a $4 \%$ em doses crescentes de 4, 5, 6, 7 e $8 \mu \mathrm{g} / \mathrm{kg} / \mathrm{min}$, com incremento da dose a intervalos de 10 minutos. Para o G2, empregou-se a mesma metodologia, substituindo-se a levomepromazina pela acepromazina, na dose de $0,1 \mathrm{mg} / \mathrm{kg}$. A eletrocardiografia e as pressões arteriais (sistólica, diastólica e média) dos animais permaneceram sob monitoramento contínuo, com início imediatamente antes da aplicação dos fármacos e término ao final do período experimental. As colheitas dos valores numéricos tiveram início imediatamente antes da aplicação dos fármacos (M1), seguidas de novas mensurações realizadas nos momentos correspondentes às doses crescentes de adrenalina $(4,5,6,7,8 \mu \mathrm{g} / \mathrm{kg} /$ minuto; M2, M3, M4, M5, M6, respectivamente). Os valores obtidos na eletrocardiografia referem o número total de batimentos ventriculares ectópicos

correspondentes às doses crescentes de adrenalina. Apenas um animal de cada grupo apresentou arritmia ventricular sustentada, sendo que o animal pré-tratado com acepromazina foi à óbito. Os resultados obtidos permitiram concluir que a levomepromazina e a acepromazina minimizam a arritmia ventricular induzida pela adrenalina, nas doses empregadas, em cães anestesiados pelo halotano. Complementarmente foi possível deduzir que o risco de óbito por uso de adrenalina em cães anestesiados com halotano é ainda menor quando se opta pelo pré-tratamento com levomepromazina.

Palavras-chave: anestesia, fenotiazinas, antiarritmicos.

SUMMARY

The aim of this work was to compare the antiarrhythmogenic effects of levomepromazine and acepromazine, in dogs anesthetized with halothane, receiving increasing doses of epinephrine. For this purpose, 19 male and female healthy adult mixed breed dogs were divided in two groups, one with $10(G 1)$ and other with 09 (G2) animals. G1 received intravenously, $1 \mathrm{mg} / \mathrm{kg}$ of levomepromazine, followed, 15 minutes later, by anesthetic induction with propofol $(5 \pm$ $1.3 \mathrm{mg} / \mathrm{kg})$. The intubation was proceeded, followed by imediate administration of halothane at 3 MAC $(2.5 \mathrm{~V} \%)$ through a semiclosed anesthetic circuit. After 50 minutes of the anesthetic induction, administration of $4 \%$ epinephrine solution had begun, with increasing doses of 4, 5, 6, 7 and $8 \mu \mathrm{g} / \mathrm{kg} / \mathrm{min}$. The increment of the doses occurred at 10 minutes intervals. For 62 the same methodology was used, except that levomepromazine was

\footnotetext{
${ }^{1}$ Pós-graduando do Curso de Doutorado em Medicina Veterinária, Área de Concentração em Cirurgia, Faculdade de Ciências Agrárias e Veterinárias (FCAV), Universidade Estadual Paulista (UNESP), Campus de Jaboticabal, SP

${ }^{2}$ Professor Assistente, Doutor, Departamento de Clínica e Cirurgia Veterinária, FCAV, UNESP, Campus de Jaboticabal, 14870-000, Jaboticabal, SP. E-mail: newton@fcav.unesp.br. Autor para correspondência.
} 
replaced with $0.1 \mathrm{mg} / \mathrm{kg}$ of acepromazine. Electrocardiography and arterial blood pressure (systolic, diastolic and mean) were continuously evaluated, starting immediately before the administration of the drugs and going on until the end of the experimental period. The measurements were made immediately before the administration of the drugs (M1), followed by new measurements related with the increasing doses of epinephrine (4, 5, 6, 7 and $8 \mu \mathrm{g} / \mathrm{kg} / \mathrm{min} ; \mathrm{M} 2, \mathrm{M3}, \mathrm{M4}, \mathrm{M5}$ and M6 respectively). The electrocardiographic values obtained were referent to the total number of ventricular premature complexes, coincident with each epinephrine dose. Only one animal of each group presented sustained ventricular arrhythmia, and the animal pretreated with acepromazine died. These results allowed the conclusion that levomepromazine and acepromazine minimizes ventricular epinephrine-induced arrhythmia in dogs anesthetized with halotane. In addiction, the risk of death by epinephrine use in dogs anesthetized with halotane and pretreated with levomepromazine seems to be even lower.

Key words: anesthesia, phenothiazines, antiarrhythmics.

\section{INTRODUÇÃO}

As arritmias cardíacas são consideradas desordens na geração dos impulsos elétricos, na sua propagação ou ambos (HOFFMAN \& CRANEFIELD, 1964). Seu desenvolvimento, muitas vezes, precipita ou intensifica uma insuficiência cardíaca, sendo que a taquicardia ventricular reduz o tempo disponível para o enchimento ventricular, diminuindo o débito cardíaco, além de aumentar a demanda de oxigênio do miocárdio (JACOBS, 1996). Algumas arritmias podem evoluir, levando a episódios de parada cardíaca e morte súbita (MUIR et al., 1999). Estudos de MATEOS et al. (1997) descreveram que as arritmias cardíacas ventriculares são as causas mais freqüientes de morte súbita, destacando-se a taquicardia ventricular e a fibrilação ventricular.

Sob anestesia geral, a arritmia ocorre, principalmente, por desequilíbrio do balanço autonômico e efeitos adversos provenientes da administração de certas drogas ou da interação entre fármacos (HOFFMAN \& CRANEFIELD, 1964).

A anestesia inalatória tornou-se técnica popular, devido, principalmente, à segurança e à facilidade de controle do plano anestésico. Porém, o halotano pode promover um aumento da automaticidade do miocárdio e conseqüentemente a propagação de impulsos provenientes de focos ectópicos, especialmente no ventrículo. Ele sensibiliza o miocárdio ou seu sistema de condução à ação arritmogênica das catecolaminas endógenas e/ou exógenas, predispondo o animal às arritmias cardíacas (KATZ \& BIGGER, 1970). O halotano possui atividade depressora sobre a freqüência cardíaca e sobre a pressão arterial (WYLLIE \& CHURCHILL-DAVIDSON， 1974). O halotano geralmente diminui a freqüência cardíaca e pode retardar a condução dos impulsos cardíacos através do nó atrioventricular e sistema de His-Purkinje, aumentando a probabilidade de ocorrência de arritmias cardíacas causadas por mecanismo de reentrada (OMOIGUI, 1998). MASSONE (1999) descreveu a ocorrência de sensibilização do miocárdio às catecolaminas quando do emprego desse anestésico volátil, podendo resultar em fibrilação e assistolia.

Estudando a dose arritmogênica da adrenalina em cães anestesiados pelo halotano e isoflurano, LEMKE et al. (1994) concluíram que as despolarizações ventriculares ectópicas advêm de dois mecanismos distintos. Um relacionado à facilitação de despolarizações ventriculares e outro, proveniente do aumento da pressão arterial, associado à diminuição da freqüência de despolarização do nódulo sinoatrial. Observaram, ainda, que a facilitação no aparecimento de extrasístoles ventriculares ocorria em momentos de elevado consumo de oxigênio.

Os fenotiazínicos também são fármacos de uso freqüente na rotina anestésica, tanto por seu efeito tranqüilizante, quanto pela potencialização de agentes anestésicos barbitúricos, não-barbitúricos e dissociativos. Produzem depressão do sistema nervoso central devido à sua ação sobre os centros nervosos inferiores, tálamo, hipotálamo e formação reticular. Apresentam, ainda, propriedades antieméticas, anti-histamínicas, anti-espasmódicas (SHORT, 1987) e, principalmente, adrenolíticas (PARADIS et al., 1959).

A levomepromazina é uma fenotiazina da "série mista", possuindo dessa forma, atividades anti-histamínica e adrenolítica acentuadas (PARADIS et al., 1959). Estudando o fármaco, NUNES et al. (1995) observaram atividade hipotensora devido à bloqueio $\alpha$-adrenérgico, dosedependente, com diminuição da regulação vasomotora. Estes efeitos impediriam a elevação da pressão arterial pela catecolamina (HOFFMAN \& LEFKOWITZ, 1991), que parece estar associada ao aparecimento de complexos prematuros ventriculares (LEMKE et al., 1994). As propriedades antiarritmogênicas da levomepromazina foram estudadas por NUNES $\boldsymbol{e t}$ al. (1999a). Os autores observaram a prevenção de complexos prematuros ventriculares em cães anestesiados pela quetamina que receberam doses crescentes de adrenalina. Eles concluíram tratar-se de fármaco de eleição na prevenção de complexos ventriculares prematuros em cães. NUNES et al. (1999b) e REZENDE $\boldsymbol{e t}$ al. (2000) confirmaram a assertiva em cães submetidos à infusão de adrenalina 
e anestesiados pelo halotano e sevoflurano, respectivamente.

Dentre os fenotiazínicos destaca-se, também, a acepromazina, que é a fenotiazina mais empregada em Medicina Veterinária. Quanto maior a sua dose, mais potente a tranqüilização e maior a hipotensão arterial. $\mathrm{Na}$ dose de $1 \mathrm{mg} / \mathrm{kg}$ por via intramuscular, o fármaco diminui a pressão arterial no cão, ocorrendo bradicardia intermitente, podendo-se observar parada sinoatrial com recuperação espontânea sem evidência de lesão cardíaca permanente (BOOTH, 1992). Segundo GLEED (1987), o débito e a freqüência cardíaca não se alteram significativamente após doses clínicas de acepromazina, entretanto, STEPIEN et al. (1995), estudando os efeitos circulatórios da acepromazina em cães, observaram que esse fármaco reduz o débito cardíaco e o volume sistólico, sem alteração da freqüência cardíaca.

A acepromazina diminui a sensibilidade do miocárdio às catecolaminas, apresentando efeito antiarritmico por ação análoga à da quinidina (LEMONICA \& PEREIRA, 1992). Seu efeito protetor contra arritmias induzidas pela adrenalina foi observado mesmo com uma dose reduzida de $0,025 \mathrm{mg} / \mathrm{kg}$ (DYSON \& PETTIFER, 1997). LIN $\boldsymbol{e}$ al. (1993) notaram que a utilização da acepromazina previamente à anestesia pela quetamina previniu o aumento das concentrações de adrenalina e noradrenalina por até duas horas após a cirurgia.

No que se refere à catecolamina, tal escolha se baseia em citações da literatura relativas à utilização da adrenalina nos estudos de arritmogenicidade, sendo sua dose arritmogênica estabelecida por TRANQUILLI et al. (1986) como aquela necessária à produção de quatro ou mais contrações prematuras ventriculares, intermitentes ou contínuas, num intervalo de 15 segundos.

Portanto, com este experimento, objetivou-se comparar as propriedades antiarritmogênicas da levomepromazina e da acepromazina em cães anestesiados pelo halotano e submetidos a doses crescentes de adrenalina.

\section{MATERIAL E MÉTODOS}

Foram utilizados 19 cães (Canis familiaris, LINNAEUS, 1758), adultos, sadios, que após terem sido selecionados, foram separados aleatoriamente, em dois grupos, sendo um de 10 (G1) e outro de 09 animais (G2).

Os animais do G1 receberam, por via intravenosa, levomepromazina ${ }^{\mathrm{a}}$, na dose de $1 \mathrm{mg} / \mathrm{kg}$, seguida 15 minutos após, pela indução anestésica geral através da administração, pela mesma via, de propofol $^{\mathrm{b}}$, na dose média de $5 \pm 1,3 \mathrm{mg} / \mathrm{kg}$. Foi procedida então a intubação orotraqueal, sendo a sonda acoplada à equipamento para administração de anestésico geral volátil em circuito valvular tipo "semi-fechado". Imediatamente foi iniciada a administração de halotano ${ }^{\mathrm{d}}$, diluido em oxigênio $(30 \mathrm{~m} \ell / \mathrm{kg} / \mathrm{min})$, na concentração de 3 CAM $(2,5 \mathrm{~V} \%)$, mensurada em analisador de gases digital ${ }^{\mathrm{e}}$. Decorridos 50 minutos do início da anestesia geral volátil, teve início a aplicação contínua, através de bomba de infusãof, por via intravenosa, de adrenalina $^{\mathrm{g}}$ a $0,04 \mathrm{mg} / \mathrm{ml}$ (4\%), diluída em solução salina a $0,9 \%$ na dose inicial de $4 \mu \mathrm{g} / \mathrm{kg} /$ minuto. A cada 10 minutos do início da administração da catecolamina, esta dose foi acrescida em $1 \mu \mathrm{g} / \mathrm{kg} /$ minuto, até a dose máxima de $8 \mu \mathrm{g} / \mathrm{kg} /$ minuto, sendo esta última dose administrada durante 10 minutos. Nos animais do G2, foi empregada a mesma metodologia, substituindo-se a levomepromazina, pela acepromazina ${ }^{\mathrm{h}}$, administrada pela mesma via, na dose de $0,1 \mathrm{mg} / \mathrm{kg}$.

A eletrocardiografia e as pressões arteriais (sistólica, diastólica e média) dos animais permaneceram sob monitoramento contínuo, com início imediatamente antes da aplicação dos fármacos e término ao final do período experimental. A eletrocardiografia foi obtida na derivação D2, através de eletrocardiógrafo digital ${ }^{\mathrm{i}}$, e a pressão arterial, através de monitor multiparamétrico , não invasivo, tipo oscilométrico, cujo manguito foi adaptado ao membro torácico direito ou esquerdo, acima da articulação úmeroradio-ulnar. As colheitas dos valores numéricos tiveram início imediatamente antes da aplicação dos fármacos (M1), seguidas de novas mensurações realizadas nos momentos correspondentes às doses crescentes de adrenalina $(4,5,6,7,8 \mu \mathrm{g} / \mathrm{kg} /$ minuto; M2, M3, M4, M5, M6, respectivamente). Os valores obtidos na eletrocardiografia referem o número total de batimentos cardíacos de origem não sinusal correspondentes às doses crescentes de adrenalina. A avaliação estatística da frequiência cardíaca e das pressões arteriais foi efetuada por meio da Análise de Perfil (MORRISON, 1967; CURI, 1980).

\section{RESULTADOS E DISCUSSÂO}

No que se refere à freqüência cardíaca, não houve diferença entre os grupos ao longo dos momentos. Observou-se, entretanto, que a freqüência cardíaca se manteve em níveis mais baixos no grupo que recebeu levomepromazina (G1) do que no grupo que recebeu acepromazina $(\mathrm{G} 2)$, embora essa diferença não tenha sido 
estatisticamente significativa. Tal comportamento pareceu mais evidente nos momentos referentes às doses mais baixas de adrenalina (4 e $5 \mu \mathrm{g} / \mathrm{kg} / \mathrm{min}$ ). $\mathrm{O}$ comportamento das curvas individuais ao longo do tempo mostrou um aumento crescente da freqüência cardíaca coincidente com o incremento das doses de adrenalina, no G1. Por outro lado, no G2, observouse um aumento marcante deste parâmetro entre o momento basal e o início da administração de adrenalina, mantendo-se posteriormente estável. Uma possível explicação para o achado poderia residir no fato de que existem diferenças significativas quanto ao potencial adrenolítico das fenotiazinas utilizadas. Neste sentido, o emprego da acepromazina pode ter determinado menor grau de bloqueio da catecolamina, o que determinou maiores médias de freqüência cardíaca (NUNES et al., 1999a), já o pré-tratamento com levomepromazina foi capaz de evitar a taquicardia, quando se empregaram doses menores de adrenalina. Complementarmente, essa fenotiazina foi responsável por manter mais elevadas as curvas de pressão arterial.

A levomepromazina não alterou a pressão arterial média, que permaneceu dentro dos padrões fisiológicos para a espécie (MUIR \& HUBBEL, 1989). No grupo que recebeu acepromazina, a pressão arterial média se manteve mais baixa, sendo a diferença entre os grupos estatisticamente significativa apenas nos momentos correspondentes a 5 e $7 \mu \mathrm{g} / \mathrm{kg} / \mathrm{min}$ de adrenalina. Tal achado sugere que o bloqueio dos receptores $\alpha$ periféricos e a depressão dos centros motores centrais promovidos pelas fenotiazinas (MUIR \& HUBBEL, 1989; BROCK, 1994) ocorreriam de forma mais marcante com a acepromazina. $\mathrm{O}$ fato da pressão arterial ter se mantido mais baixa nesse grupo, acrescido do que já foi discutido para a frequiência cardíaca, poderia ser responsável pela frequiência cardíaca aumentada. Cabe lembrar que esse aumento poderia ser compensatório, buscando a manutenção do débito cardíaco (MUIR \& HUBBEL, 1989; BROCK, 1994).

A pressão arterial diastólica apresentou comportamento idêntico ao da pressão arterial média. A pressão arterial sistólica também evoluiu de maneira semelhante à média, mas diferiu significativamente entre grupos apenas no momento referente a $7 \mu \mathrm{g} / \mathrm{kg} / \mathrm{min}$ de adrenalina. Isto posto, parece evidente que a discussão da pressão arterial média espelha aquela que se poderia redigir, relativamente às demais. (Tabela 1 ).

No grupo pré-tratado com levomepromazina, observou-se a presença de 1 batimento ectópico ventricular em dois cães, tendo este evento ocorrido em M3 $(5 \mu \mathrm{g} / \mathrm{kg} / \mathrm{min})$ para um animal e em M4 $(6 \mu \mathrm{g} / \mathrm{kg} / \mathrm{min})$ para o outro. Apesar desse fato, os batimentos ventriculares ectópicos, encontraram-se em nível inferior ao estabelecido por TRANQUILLI $\boldsymbol{e}$ t al. (1986) como característico de arritmia em cães. É importante destacar que apenas um animal deste grupo apresentou arritmia ventricular sustentada, a qual ocorreu nos dois últimos momentos (7 e $8 \mu \mathrm{g} / \mathrm{kg} / \mathrm{min})$, onde foram observados 108 e 116 batimentos ventriculares ectópicos, respectivamente. Chama a atenção o fato de que apesar do padrão de taquicardia ventricular multiforme apresentado, essa não evoluiu significativamente a ponto de induzir a fibrilação ventricular, mesmo sob continuado incremento das doses de adrenalina, permitindo a conclusão do período experimental.

No grupo que recebeu acepromazina, observou-se a presença de 1 batimento de origem ectópica ventricular em três cães, neste caso ocorrendo em M3 ( $5 \mu \mathrm{g} / \mathrm{kg} / \mathrm{min})$, M5 ( $7 \mu \mathrm{g} / \mathrm{kg} / \mathrm{min})$ e M6 $(8 \mu \mathrm{g} / \mathrm{kg} / \mathrm{min})$ respectivamente. Houve também um animal que apresentou taquicardia ventricular multiforme, a qual ocorreu no momento correspondente a $6 \mu \mathrm{g} / \mathrm{kg} / \mathrm{min}$ (M4). Nesse caso, ao contrário do descrito para o grupo anterior, a arritmia surgiu repentinamente e evoluiu rapidamente para fibrilação ventricular. Apesar de ter-se cessado imediatamente a administração de adrenalina e de halotano, e ter-se realizado lavagem pulmonar com alto fluxo de $\mathrm{O}_{2}$, além da técnica de ressuscitação cardiorrespiratória (MILLER, 1989), incluindo cardioversão elétrica a $5 \mathrm{~J} / \mathrm{kg}$, não foi possível reverter o quadro e o animal foi à óbito. Para a análise estatística proposta, esse animal precisou ser descartado, permanecendo o G2 com 9 animais.

Face as altas doses de adrenalina empregadas e a despeito dos dois casos de arritmia ventricular observados, destaca-se a utilidade dos agentes $\alpha$-bloqueadores na proteção do miocárdio contra irregularidades cardíacas induzidas pela adrenalina (MUIR \& HUBBEL, 1989; LEMONICA \& PEREIRA, 1992; DYSON \& PETTIFER, 1997; LEMKE, 1999; NUNES $\boldsymbol{e}$ t al., 1999a; NUNES $\boldsymbol{e}$ al., 1999b; REZENDE $\boldsymbol{e t}$ al., 2000). Tais efeitos poderiam ser explicados pela prevenção da elevação da pressão arterial, promovida pela catecolamina (HOFFMAN \& LEFKOWITZ, 1991), mecanismo que, em parte, atua no desenvolvimento de complexos prematuros ventriculares (LEMKE $\boldsymbol{e t}$ al., 1994). 
Tabela 1 - Efeitos do pré-tratamento com levomepromazina (G1) e acepromazina $(\mathrm{G} 2)$ na freqüência cardíaca, pressão arterial sistólica, média e diastólica de cães anestesiados pelo halotano e submetidos a doses crescentes de adrenalina.

\begin{tabular}{|c|c|c|c|c|c|c|c|}
\hline Variáveis & & M1 Basal & $\begin{array}{c}\mathrm{M} 2 \\
4 \mu \mathrm{g} / \mathrm{kg} / \mathrm{min}\end{array}$ & $\begin{array}{c}\mathrm{M} 3 \\
5 \mu \mathrm{g} / \mathrm{kg} / \mathrm{min}\end{array}$ & $\begin{array}{c}\mathrm{M} 4 \\
6 \mu \mathrm{g} / \mathrm{kg} / \mathrm{min}\end{array}$ & $\begin{array}{c}\text { M5 } \\
7 \mu \mathrm{g} / \mathrm{kg} / \mathrm{min}\end{array}$ & $\begin{array}{c}\text { M6 } \\
8 \mu \mathrm{g} / \mathrm{kg} / \mathrm{min}\end{array}$ \\
\hline Freqüência cardíaca & G1 G2 & $\begin{array}{l}110 \pm 12^{*} \\
104 \pm 20^{*}\end{array}$ & $\begin{array}{l}140 \pm 47^{*} \\
172 \pm 25^{*}\end{array}$ & $\begin{array}{l}156 \pm 43^{*} \\
182 \pm 23^{*}\end{array}$ & $\begin{array}{l}170 \pm 41^{*} \\
187 \pm 22^{*}\end{array}$ & $\begin{array}{l}188 \pm 30^{*} \\
195 \pm 22^{*}\end{array}$ & $\begin{array}{l}190 \pm 28^{*} \\
197 \pm 19^{*}\end{array}$ \\
\hline Pressão arterial sistólica & G1 G2 & $\begin{array}{c}119,9 \pm 13,5 \\
112,1 \pm 8,2\end{array}$ & $\begin{array}{l}131,8 \pm 13,5 \\
115,7 \pm 26,5\end{array}$ & $\begin{array}{c}131,7 \pm 21,3 \\
111 \pm 23,5\end{array}$ & $\begin{array}{l}131,2 \pm 18,6 \\
123,2 \pm 17,7\end{array}$ & $\begin{array}{c}136,3 \pm 10,7^{\bullet} \\
118 \pm 24,5^{\bullet}\end{array}$ & $\begin{array}{l}127,3 \pm 16,6 \\
129,4 \pm 22,4\end{array}$ \\
\hline Pressão arterial média & G1 G2 & $\begin{array}{l}84,8 \pm 7,5 \\
87,1 \pm 8,9\end{array}$ & $\begin{array}{c}89,4 \pm 9,1 \\
79,5 \pm 17,7\end{array}$ & $\begin{array}{l}91,4 \pm 14,5^{\circ} \\
73,4 \pm 16,5^{\circ}\end{array}$ & $\begin{array}{l}92,2 \pm 13,5 \\
83,5 \pm 14,2\end{array}$ & $\begin{array}{c}95,8 \pm 8,6^{\circ} \\
80,8 \pm 17,2^{\circ}\end{array}$ & $\begin{array}{c}89.2 \pm 12,5 \\
89 \pm 17,2\end{array}$ \\
\hline Pressão arterial diastólica & G1 G2 & $\begin{array}{c}65,9 \pm 6,9 \\
68,3 \pm 10,5\end{array}$ & $\begin{array}{c}65,5 \pm 7,5 \\
59,7 \pm 12,3\end{array}$ & $\begin{array}{c}68,2 \pm 10,1^{\bullet} \\
53 \pm 11,2^{\bullet}\end{array}$ & $\begin{array}{l}68,5 \pm 10,3 \\
60,6 \pm 11,6\end{array}$ & $\begin{array}{c}71,5 \pm 8,3 \\
59,6 \pm 12,1\end{array}$ & $\begin{array}{c}67,4 \pm 9,0 \\
66,3 \pm 14,8\end{array}$ \\
\hline
\end{tabular}

Valores expressos em média \pm desvio padrão.

*Diferenças entre os momentos dentro de cada grupo.

-Diferenças entre os grupos ao longo dos momentos.

\section{CONCLUSÃO}

Frente aos resultados obtidos, pode-se concluir que a levomepromazina e a acepromazina minimizam a arritmia ventricular induzida pela adrenalina, nas doses de $4,5,6,7,8 \mu \mathrm{g} / \mathrm{kg} /$ minuto, em cães anestesiados pelo halotano. Complementarmente pode-se afirmar que o risco de óbito por uso de adrenalina em cães anestesiados com halotano é ainda menor quando se opta pelo pré-tratamento com levomepromazina.

\section{FONTES DE AQUISIÇÃO}

${ }^{a}$ Neozine - Rhodia S.A. Divisão Farmacêutica

${ }^{\mathrm{b}}$ Diprivan - Zeneca Farmacêutica do Brasil Ltda.

${ }^{c}$ Ohmeda - mod. Excel 210SE

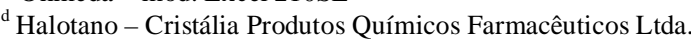

${ }^{\mathrm{e}}$ Ohmeda - mod. 5220

${ }^{\mathrm{f}}$ Lifemed - mod. FARS 600

${ }^{\mathrm{g}}$ Cloridrato de adrenalina 1/1000 - Laboratório Santista Ltda.

${ }^{\mathrm{h}}$ Acepran - Univet S.A. Indústria Veterinária

${ }^{i}$ TEB mod.ECG-PC software versão 1.10

${ }^{\mathrm{j}}$ Digimax 5000 - mod. ESFMN 2T.

\section{AGRADECIMENTOS}

Agradecemos à Fundação de Amparo à Pesquisa do Estado de São Paulo - FAPESP pelo apoio financeiro.

\section{REFERÊNCIAS BIBLIOGRÁFICAS}

BOOTH. N. H. Agentes psicotrópicos. In: BOOTH, N. H., McDONALD, L. E. Farmacologia e terapêutica em veterinária. 6.ed. Rio de Janeiro : Guanabara Koogan, 1992. p.289-314

BROCK, N. Acepromazine revisited. Can Vet J, v.35, p.458$459,1994$.
CURI, P.R. Análise de medidas repetidas em experimentos biológicos. Rev Bras Estat, v.41, n.161, p.137-150. 1980.

DYSON, D.H., PETTIFER, G.R. Evaluation of the arrhythmogenicity of o low dose of acepromazine: comparison with xylazine. Can J Vet Res, v.61, n.4, p.241245, 1997.

GLEED, R.D. Tranquilizers and sedatives. In: SHORT, C.E. Principles \& practice of veterinary anesthesia. Baltimore : Williams \& Wilkins, 1987. p.16-27.

HOFFMAN, B.F., CRANEFIELD, P.F. The physiological basis of cardiac arrhytmias. Am J Med, v.37, p.670-684, 1964.

HOFFMAN, B.B., LEFKOWITZ, R.J. Catecolaminas e drogas simpatomiméticas. In: GILMAN, A.G., RALL, T.W., NIES, A.S., et al. Goodman \& Gilman: as bases farmacológicas da terapêutica. 8.ed. Rio de Janeiro : Guanabara, 1991. p.123-143.

JACOBS, G.J. Cardiomyopathies: their classification and pathophysiologic features. Vet Med, v.91, n.5, p.436-444, 1996.

KATZ, R.L., BIGGER, J.T. Cardiac arrhytmia during anesthesia and operation. Anestesiology, v.33, p.193-213, 1970.

LEMKE, K.A., TRANQUILLI, W.J., THURMON, J.C., $\boldsymbol{e}$ t al. Influence of cholinergic blockade on the development of epinephrine-induced ventricular arrythmias in halothane and isoflurane-anesthetized dogs. Vet Surg, v.23, p.61-66, 1994.

LEMKE, K.A. Pharmacology. In: THURMON, J.C., TRANQUILLI, W.J., BENSON, G.J. Essentials of small animal anesthesia \& analgesia. Baltimore : Williams and Wilkins, 1999. 580p.

LEMONICA, L., PEREIRA, S.M. Medicação pré-anestésica. In: CASTIGLIA, Y.M.M. Temas de anestesiologia para uso de graduação em medicina. São Paulo: UNESP, 1992. p.27-38.

LIN, H.C., BENSON, G.J., THURMON, J.C., et al. Influence of anesthetic regimens on the perioperative catecholamine responses associated with onychectomy in cats. Am J Vet Res, v.54, n.10, p.1721-1724, 1993. 
MASSONE, F. Anestesiologia veterinária. Farmacologia e técnicas. 3.ed. Rio de Janeiro : Guanabara Koogan, 1999. 252 p.

MATEOS, J.C.P., KORMANN, D.S., MATEOS, E.I.P. A terapêutica antiarrítmica previne a morte súbita? Rev Soc Cardiol Est São Paulo, v.7, n.2, p.240-254, 1997.

MILLER, R.D. Tratado de anestesia. 2.ed. São Paulo : Manole, 1989. 2525p

MORRISON, D.F. Multivariate statistical methods. New York : MaC Grows Hill Book, 1967. 388p.

MUIR, W.W., HUBBELL, J.A.E. Handbook of veterinary anesthesia. Toronto : Mosby, 1989. 339p.

MUIR, W.W., SAMS, R.A., MOISE, N.S. Pharmacology and pharmacokinetics of antiarrhythmic drugs. In: FOX, P.R., SISSON, D., MOISE, N.S. Textbook of canine and feline cardiology. 2.ed. Philadelphia : Saunders, 1999. Cap.17, p.307-330.

NUNES, N., POMPERMAYER, L.G., PIROLO, J., et al. Emprego do metaraminol no bloqueio da hipotensão produzida pela levomepromazina em cães. Braz J Vet Res An Sci, v.32, n.2, p.120-124, 1995.

NUNES, N., MASSONE, F., POMPERMAYER, L.G., et al. Estudo da atividade antiarritmogênica da levomepromazina em cães submetidos a anestesia pela quetamina. Ciência Rural, v.29, n.2, p.291-295, 1999a.
NUNES, N., SANTOS, P.S.P., REZENDE, M.L. et al. Emprego da levomepromazina no bloqueio da arritmia cardíaca induzida pela adrenalina, em cães anestesiados pelo halotano. Ars Vet, v.15, n.3, p.164-169, 1999b.

OMOIGUI, S. Manual de drogas usadas em anestesia. 2.ed. São Paulo : Santos, 1998. 566p.

PARADIS, B., PLANTE, G., AUBUT, J.A. La levomepromazine (Nozinam ou 7044 RP). Laval Med, v.38, p.433-447, 1959.

REZENDE, M.L., SANTOS, P.S.P., NUNES, N., et al Estudo da atividade antiarritmogênica da levomepromazina, em cães anestesiados pelo sevoflurano. Ciência Rural, v.30, n.3, p.421-424, 2000 .

SHORT, C.E. Principles \& pratice in veterinary anesthesia. Baltimore : Willian \& Wilkins, 1987. 669p.

STEPIEN, R.L., BONAGURA, J.D., BEDNARSKI, R.M., $\boldsymbol{e}$ t al. Cardiorespiratory effects of acepromazine maleate and buprenorphine hydrochloride in clinically normal dogs. American Journal of Veterinary Research, v.56, n.1, p.7884, 1995.

TRANQUILLI, W.J., THURMON, J.C., BENSON, G.J. et al Alteration in the arrythmogenic dose of epinephrine (ADE) following xylazine administration to halothane-anesthetized dogs. J Vet Pharmacol Therap, v.9, n.2, p.198-203, 1986.

WYLLIE, W.D., CHURCHIL-DAVIDSON, H.C. Anestesiologia. 3.ed. Rio de Janeiro: Guanabara,1974. $1031 \mathrm{p}$. 